\title{
Impact of Air Pollution on Carotenoid and Chlorophyll Contents in Three Forest Reserves in Edo State, Nigeria
}

\author{
Ogboru R.O ${ }^{1}$, Okolie L.P ${ }^{2}$, Idibie C. A \\ ${ }^{1}$ Forestry Research Institute of Nigeria:Moist Forest Research Station, P.M.B 2444, Benin-City \\ ${ }^{2}$ Delta State University Abraka, Delta State, Nigeria.P.M.B 1, Abraka, Nigeria \\ ${ }^{3}$ Michael and Cecilia Ibru University, Agbarha-Otor, Ughelli, Delta State, Nigeria
}

\begin{abstract}
Evaluation of the contents levels of chlorophylla, chlorophyll b and Carotenoid, which are essential plant compounds that convert light energy into chemical energy that powers the biosphere, was carried out in terms of their responses to ambient air pollution. This involved using ten species from three different locations(Ogba, UNIBEN and Saponba Forest Reserves) over a selected period of time. Results showed that Termilinacatapa had the highest value of Chlorophyll-a content with a value of $36.49 \mathrm{mg} / 100 \mathrm{~g}$ in Ogba Forest Reserve while the lowest value was also in Terminilinacatapa with a value of $0.55 \mathrm{mg} / 100 \mathrm{~g}$ in UNIBEN Forest Reserve. Chlorophyllbcontent was highest in Tectonagrandis in the Ogba Forest Reserve of a value of $84.66 \mathrm{mg} / 100 \mathrm{~g}$ and lowest with a value of $84.66 \mathrm{mg} / 100 \mathrm{~g}$ and the lowest with a value $4.15 \mathrm{mg} / 100 \mathrm{~g}$ in Naucleadiderrichi also in the Ogba Forest Reserve. Carotenoid(x+c) content was highest in Termilinacatapa with a value of $36.49 \mathrm{mg} / 100 \mathrm{~g}$ and Lowest in UNIBEN Forest Reserve with a value of 0.55mg/100g. Results also showed variations in contents values tend to be far more of a negative variance than positive, which indicates environmental pollution; particularly air pollution is a major cause of biomarkers reduction in plants where UNIBEN Forest Reserve experienced the highest effect.
\end{abstract}

Keywords: Chlorophyll-a, Chlorophyll-b, Carotenoids, Photosynthetic Pigments, Forest Reserve

\section{Introduction}

Chlorophylls and carotenoids are essential '[pigments of higher plant assimilatory tissues and are responsible for variations of colour from dark green to yellow. Moreover, they also play important roles in photosynthesis capturing light energy which is converted into chemical energy (Bauernfeind, 1981; Young and Britton, 1993). Through the process of photosynthesis, chlorophyll has the ability of channeling the radiant energy of sunlight into chemical energy of organic carbon compounds in the cell(Nichiporovich, 1974). Carotenoids are a class of natural fat soluble pigments found inplants, algae and photosynthetic bacteria, where they also play a critical role in the photosynthetic process and also protect chlorophyll from photoxidative destruction (Siefermann-Harms, 1987). Total leaf pigments include chlorophyll-a, chlorophyll-b and carotenoids, which are necessary for photosynthetic process. The content of foliar pigments varies depending on the species. Variation of leaf pigment(chlorophylls and carotenoids) and its relation can be due to internal factors and environmental conditions. The ration of chlorophyll-a and chlorophyll-b in terrestrial plants has been used as an indicator of response to light shade conditions (Porra, 1991; Vicaset al., 2010). The intensity of colour and its shade is also due to the level of chlorophyll pigments and their proportions. (Lisiewskaet al., 2001). The constituents of chlorophyll $\mathrm{a} / \mathrm{b}$ are considered sensitive biomarker of pollution and environmental stress (Tripathi\&Gautam, 2007). The absorbance properties of these pigments facilitate the qualitative and quantitative analysis of them (James \&Akaranta, 2011).
In higher plants, Chlorophyll-a and Chlorophyll-b are actually the most forms of chlorophyll and are essential for the conversion of light energy into stored chemical energy that powers the biosphere (Richardson et al.,2002). Chlorophyll-c and Chlorophyll-d are other common forms of chlorophylls, but are only found in photosynthetic members of the Chromista and Dinoflagellates (Ceulemans\&Saugier 1993) The ratio of Chlorophyll and carotenoid pigment is strongly affiliated with photosynthetic functioning of vegetation (Gamonet al., 1997) and this capacity varies in a range of environmental conditions. Periods of medium to long term stress can often be detected by decreases in chlorophyll content (Lincenthaler 2001; Zarco-Tejada et al., 2002), while short-term changes can be detected via carotenoid metabolism(Demmig-Adams 1996)

Artificial forests are usually located from boreal to tropical zones and involves indigenous or introduced tree species, but exotic species are more common in tropical plantations grown for timber production or rural development and cover about 88 million hectares (Evans \& Turnbull 2004). Artificial forests differ from natural forests because they consist both of indigenous and non-indigenous tree species which differs in form, composition and intensity of management (RuizJaen et al., 2005).This study is aimed at evaluating the levels of chlorophyll-a, Chlorophyll-b and Carotenoid content in 3 artificial forests in Edo state( Ogba, UNIBEN and Saponba Forest Reserves), Nigeria from selected months in the raining season till dry season in order to access their response to ambient air pollutants. The Objectives of this study was to investigate if there is significant difference between chlorophyll-a, chlorophyll-b and carotenoids within ten tree species and three locations within the study period 


\section{International Journal of Science and Research (IJSR) \\ ISSN (Online): 2319-7064}

Index Copernicus Value (2013): 6.14 | Impact Factor (2014): 5.611

and also show with chart the trend of values analyzed for within the study period.

\section{Methodology}

\section{Study Area}

The study sites are

1) Saponba lies between latitude $06^{0} \quad 25,32 \% \mathrm{~N}$ and longitude $05^{0} 5^{\prime} 28^{\prime \prime} \mathrm{E}$ and has an area of $521 \mathrm{~km}^{2}$ in the rain forest zone. The forest reserve has two main rainfall seasons, the rainy and dry seasons. The annual rainfall ranges from $2056 \mathrm{~mm}$ to $2332 \mathrm{~mm}$. The temperature ranges from a minimum of $21.6^{0 \mathrm{C}}$ to a maximum of $31^{\mathrm{OC}}$. The soils are acidic, leached, well drained, predominantly loamy sand in texture. Rain forest, moist semi-deciduos (FRIN, 1977). The red soil is fertile and many of the people farm crops such as yam, cassava and beans, or cash crops including oil palm, cocoa and rubber.

2) Uniben Forest Reserve: The Uniben study site is located within the eastern part of the main campus. The site falls within Latitude $6^{0} 24^{1} 20.9^{11} \mathrm{~N}$ and Longitude $5^{0} 38^{1} 52^{11} \mathrm{E}$. The reserve lies between the Ikpoba River and the Benin Auchi Road. It is described as a typical rain forest zone, rainfall is usually high, above $2000 \mathrm{~mm}$ annually and in some cases exceeding $8000 \mathrm{~mm}$ annually. University of Benin posseses an annual rainfall of $1500 \mathrm{~mm}$ (Uniben master plan, 1993) with an average temperature of $27^{\circ} \mathrm{C}$. The topography of the land is a gentle slope which falls eastwards, while its geology consist of relatively thick layer of sedimentary sands and sandy-clays of tertiary age. The soil of the sites varies from rhodicpaleduts to typical paleduts under current United States department of Agriculture. U.S.D.A classification (Usuanlele, 1999)

3) Ogba Forest Reserve: Ogba Forest Reserve is located in of Egor Local government area of Edo state, Nigeria. The reserve lies between Longitude $6^{0} 19^{1} \mathrm{~N}$ and Latitude $5^{0}$ $41^{1} \mathrm{E}$. It is situated $7 \mathrm{~km}$ south-west of Benin-City. It is regarded as a tropical of sub tropical moist broad leaf forest. Thereis a presence of OgbaRiver within the forest.(Iyawe 1989)

\section{Plants}

The Ten (10) Forest trees which were found in these three artificial forest reserves in Edo state, Nigeria and used for this study are listed below in the table:

\begin{tabular}{|c|c|c|}
\hline Species no & Scientific names & Common names \\
\hline 1 & Naucleadiderrichi & Opepe \\
\hline 2 & Terminaliacatapa & Almond \\
\hline 3 & Gmelinaaborea & Beechwood \\
\hline 4 & Khayagradifiolia & African mahoghany \\
\hline 5 & Tectonagrandis & Teak \\
\hline 6 & Gambayaalbidum & Cherry /African star apple \\
\hline 7 & Cedrelaodorota & Cigar box tree \\
\hline 8 & Triplochitonscleroxylon & Obeche \\
\hline 9 & Terminaliaivorensis & Black afara \\
\hline 10 & Garcina kola & Bitter kola \\
\hline
\end{tabular}

\section{Sample Collection}

Samples were collected in triplicates in the morning hours five meters above the ground from twenty tree species for four (4) months (September 2014-till January, 2015), 2 months during the raining season and 2 months in the dry season. Each week analysis was done for one forest location every month to minimize errors. These samples were immediately brought to the laboratory in a polythene bag for further analysis.

\section{Determination of Total Chlorophyll and Carotenoid Content}

This was done according to the method also adopted also by Agbaire and Esiefarienrhe (2009); Lichtentaler (1985) and Wellburn(1994). $3 \mathrm{~g}$ of the fresh leaves samples of these trees species was weighed in triplicates and blended and extracted with $10 \mathrm{ml}$ of $80 \%$ acetone each. It was left for 15 minutes for thorough extraction. The liquid portion was filtered into another test tube and will be centrifuged at $2500 \mathrm{pm}$ for three minutes each. The supernatant was collected and the absorbance was taken at $645 \mathrm{~nm}$ and $662 \mathrm{~nm}$ for chlorophyll a and band 470nm for carotenoids respectively using a spectrophotometer with Model 752

Equations used for calculation are presented below:

Chlorophyll-a $=11.75 \mathrm{~A} 662-2.350 \mathrm{~A} 645$

Chlorophyll-b = 18.61-3.960A662

Carotene $=1000 \mathrm{~A} 470-2.270 \mathrm{Chl}$ a-81.4Chlb/227

Measurements were done in triplicates.

\section{Results and Discussions}

The results of chlorophyll and carotenoid concentrations in 10 tree species analyzed for between September 2014 and January, 2015, for this study are presented below in Table 1Table 9

Table 1: Chlorophyll a(Ch a)values of tree species from September, 2014 to January 2015 for Ogba Forest Reserve.

\begin{tabular}{|c|c|c|c|c|c|c|}
\hline S/N & $\begin{array}{c}\text { CHLOROPHYLL } \\
a(\text { Ch a }) \\
\text { Tree Species }\end{array}$ & $\begin{array}{c}12^{\text {th }} \\
\text { SEPT }\end{array}$ & $\begin{array}{c}13^{\text {th }} \\
\text { OCT }\end{array}$ & $2^{\text {nd }}$ & $13^{\text {th } J}$ & \\
& DEC & AN & AVR \\
\hline 1 & Naucleadiderichi & 8.40 & 21.02 & 15.66 & 14.25 & 14.83 \\
\hline 2 & Terminaliacatapa & 42.91 & 40.68 & 32.51 & 29.84 & 36.49 \\
\hline 3 & Gmelinaaborea & 4.22 & 3.87 & 3.26 & 3.73 & 3.77 \\
\hline 4 & Khayagrandiofolia & 20.76 & 41.40 & 34.69 & 35.54 & 33.10 \\
\hline 5 & Tectonagrandis & 29.03 & 35.83 & 23.72 & 22.95 & 27.88 \\
\hline 6 & Gambayaalbidum & 19.01 & 32.68 & 19.33 & 17.90 & 22.23 \\
\hline 7 & Cedralaodurata & 16.89 & 18.25 & 13.76 & 13.68 & 15.65 \\
\hline 8 & Triploclantinscherox & & & & & \\
& ylon & 22.63 & 19.47 & 16.12 & 15.80 & 18.51 \\
\hline 9 & Termilinaivorensis & 6.06 & 7.69 & 3.08 & 2.11 & 4.74 \\
\hline 10 & Garcina kola & 12.75 & 3.80 & 2.83 & 1.97 & 5.34 \\
\hline
\end{tabular}




\section{International Journal of Science and Research (IJSR) \\ ISSN (Online): 2319-7064}

Index Copernicus Value (2013): 6.14 | Impact Factor (2014): 5.611

Table 2: Chlorophyll a (Ch a)values of tree species from September, 2014to January 2015 for Uniben Forest Reserve.

\begin{tabular}{|c|c|c|c|c|c|c|}
\hline S/N & $\begin{array}{c}\text { CHLOROPHYLLa } \\
(\text { Ch-a) Trees Species }\end{array}$ & $19^{\text {th }}$ SEPT & $20^{\text {th }}$ OCT & $8^{\text {th }}$ DEC & $20^{\text {th }}$ JAN & AVR \\
\hline 1 & Naucleadiderrichi & 1.07 & 2.56 & 2.09 & 2.04 & 1.94 \\
\hline 2 & Terminaliacatapa & -1.42 & 1.73 & 1.45 & 0.43 & 0.55 \\
\hline 3 & Gmelinaaborea & 5.95 & 2.42 & 1.73 & 2.37 & 3.12 \\
\hline 4 & Khayagrandiofolia & 10.05 & 3.61 & 3.55 & 4.27 & 5.37 \\
\hline 5 & Tectonagrandis & 100.21 & 10.95 & 10.40 & -3.33 & 29.56 \\
\hline 6 & Gambayaalbidum & 76.13 & -2.40 & -2.28 & -1.30 & 17.54 \\
\hline 7 & Cedralaodurata & 6.33 & 2.28 & 1.26 & 1.03 & 2.72 \\
\hline 8 & Triploclantinscheroxylon & 3.90 & 3.15 & 3.11 & 2.20 & 3.09 \\
\hline 9 & Termilinaivorensis & 23.97 & 7.95 & 6.35 & -0.33 & 9.49 \\
\hline 10 & Garcina kola & 14.59 & 2.10 & 1.00 & -0.68 & 4.25 \\
\hline
\end{tabular}

Table 3: Chlorophyll a(Ch a)values of tree species from

September, 2014 to January 2015 from SakponbaForest Reserve.

\begin{tabular}{|c|c|c|c|c|c|c|}
\hline S/N & $\begin{array}{c}\text { CHLOROPHYLL A(Ch-a) } \\
\text { Trees Species }\end{array}$ & $\mathbf{2 6}^{\text {th }} \mathbf{S E P T}$ & $\mathbf{2 7}^{\text {th }} \mathbf{O C T}$ & $\mathbf{1 6}^{\text {th }} \mathbf{D E C}$ & $\mathbf{2 7}^{\text {th }}$ JAN & AVR \\
\hline 1 & Naucleadiderrichi & 9.84 & -1.12 & -0.94 & -0.57 & 1.80 \\
\hline 2 & Terminaliacatapa & 19.64 & 0.38 & 0.52 & 0.39 & 5.23 \\
\hline 3 & Gmelinaaborea & 10.57 & 3.33 & 3.82 & 3.87 & 5.40 \\
\hline 4 & Khayagrandiofolia & 31.12 & 3.59 & 3.73 & 4.11 & 10.63 \\
\hline 5 & Tectonagrandis & 46.97 & 13.84 & 14.28 & 9.97 & 21.26 \\
\hline 6 & Gambayaalbidum & 32.17 & 11.35 & 10.86 & 14.61 & 17.25 \\
\hline 7 & Cedralaodurata & 25.42 & 18.48 & 17.52 & 15.45 & 19.22 \\
\hline 8 & Triploclantinscheroxylon & 1.39 & 1.91 & 1.20 & 0.85 & 1.34 \\
\hline 9 & Termilinaivorensis & -10.56 & 14.69 & 13.99 & 13.05 & 7.79 \\
\hline 10 & Garcina kola & 2.29 & 2.10 & 0.89 & 0.71 & 1.50 \\
\hline
\end{tabular}

Table 4: Chlorophyll B(Ch b) values of tree species from

September, 2014 to January 2015 from OgbaForest Reserve

\begin{tabular}{|c|c|c|c|c|c|c|}
\hline S/N & $\begin{array}{c}\text { CHLOROPHYLL B }(\mathbf{C h} \text { b) } \\
\text { Trees Species }\end{array}$ & 12th SEPT & 13th OCT & 2nd DEC & 13th JAN & AVR \\
\hline 1 & Naucleadiderrichi & 11.93 & -0.10 & 2.76 & 2.02 & 4.15 \\
\hline 2 & Terminaliacatapa & 19.30 & 35.81 & 34.19 & 31.44 & 30.18 \\
\hline 3 & Gmelinaaborea & 19.28 & 20.58 & 17.83 & 14.63 & 18.08 \\
\hline 4 & Khayagrandiofolia & 34.53 & 27.52 & 22.03 & 17.62 & 25.43 \\
\hline 5 & Tectonagrandis & 20.92 & 114.35 & 108.48 & 94.92 & 84.66 \\
\hline 6 & Gambayaalbidum & 14.73 & 28.90 & 23.43 & 20.36 & 21.86 \\
\hline 7 & Cedralaodurata & 21.57 & 22.31 & 17.49 & 13.57 & 18.74 \\
\hline 8 & Triploclantinscheroxylon & 16.59 & 26.22 & 21.03 & 16.74 & 20.15 \\
\hline 9 & Termilinaivorensis & 22.50 & 26.39 & 24.56 & 21.26 & 23.68 \\
\hline 10 & Garcina kola & 23.93 & 28.75 & 26.69 & 24.05 & 25.85 \\
\hline
\end{tabular}

Table 5: Chlorophyll B(Ch-b)values of tree species from September, 2014 to January 2015 from UnibenForest Reserve

\begin{tabular}{|l|l|r|r|r|r|r|}
\hline $\mathrm{S} / \mathrm{N}$ & $\begin{array}{l}\text { CHLOROPHYLL B (Ch-b) } \\
\text { Trees Species }\end{array}$ & $19^{\text {th }}$ SEPT & $20^{\text {th }}$ OCT & $8^{\text {th }}$ DEC & $\begin{array}{r}20^{\text {th }} \\
\text { JAN }\end{array}$ & AVR \\
\hline 1 & Naucleadiderrichi & 19.82 & 12.12 & 9.22 & 6.40 & 11.89 \\
\hline 2 & Terminaliacatapa & 23.56 & 18.98 & 15.94 & 12.72 & 17.80 \\
\hline 3 & Gmelinaaborea & 22.58 & 11.33 & 10.27 & 4.87 & 12.26 \\
\hline 4 & Khayagrandiofolia & 27.30 & 13.22 & 9.86 & 3.26 & 13.41 \\
\hline 5 & Tectonagrandis & 72.70 & 17.83 & 14.46 & 12.18 & 29.29 \\
\hline 6 & Gambayaalbidum & 19.25 & 26.08 & 22.57 & 15.35 & 20.81 \\
\hline 7 & Cedralaodurata & 49.87 & 16.48 & 13.85 & 9.72 & 22.48 \\
\hline 8 & Triploclantinscheroxylon & 19.76 & 12.60 & 9.33 & 6.46 & 12.04 \\
\hline 9 & Termilinaivorensis & 48.71 & 22.76 & 22.06 & 14.72 & 27.06 \\
\hline 10 & Garcina kola & 21.16 & 15.37 & 14.04 & 13.85 & 16.11 \\
\hline
\end{tabular}

Volume 5 Issue 1, January 2016 www.ijsr.net 


\section{International Journal of Science and Research (IJSR) \\ ISSN (Online): 2319-7064 \\ Index Copernicus Value (2013): 6.14 | Impact Factor (2014): 5.611}

Table 6: Chlorophyll- b(Ch-b)values of tree species $(\mathrm{mg} / 100 \mathrm{~g})$ from September, 2014 to January 2015 from SakponbaForest Reserve

\begin{tabular}{|c|c|c|c|c|c|c|}
\hline S/N & $\begin{array}{c}\text { CHLOROPHYLL B(Ch-b) } \\
\text { Trees Species }\end{array}$ & $26^{\text {th }}$ SEPT & $27^{\text {th }}$ OCT & $16^{\text {th }}$ DEC & $27^{\text {th }}$ JAN & AVR \\
\hline 1 & Naucleadiderrichi & 19.86 & 17.87 & 13.97 & 10.52 & 15.56 \\
\hline 2 & Terminaliacatapa & 21.42 & 22.13 & 15.92 & 12.65 & 18.03 \\
\hline 3 & Gmelinaaborea & 14.00 & 12.31 & 7.87 & 4.88 & 9.77 \\
\hline 4 & Khayagrandiofolia & 27.89 & 30.04 & 23.16 & 18.34 & 24.86 \\
\hline 5 & Tectonagrandis & 24.19 & 24.69 & 20.42 & 14.91 & 21.05 \\
\hline 6 & Gambayaalbidum & 18.97 & 17.61 & 15.36 & 14.69 & 16.66 \\
\hline 7 & Cedralaodurata & 19.85 & 22.35 & 16.25 & 11.82 & 17.57 \\
\hline 8 & Triploclantinscheroxylon & 18.16 & 14.64 & 11.68 & 8.90 & 13.34 \\
\hline 9 & Termilinaivorensis & 70.33 & 17.51 & 14.10 & 9.36 & 27.83 \\
\hline 10 & Garcina kola & 66.68 & 12.06 & 11.14 & 9.70 & 24.89 \\
\hline
\end{tabular}

Table 7: Carotenoid $(\mathrm{x}+\mathrm{c})$ values of tree species, $(\mathrm{mg} / 100 \mathrm{~g})$ from September, 2014 to January 2015 from OgbaForest Reserve

\begin{tabular}{|c|c|c|c|c|c|c|}
\hline S/N & $\begin{array}{c}\text { CAROTENOIDS }(x+c) \\
\text { Trees Species }\end{array}$ & $\begin{array}{c}12^{\text {th }} \\
\text { SEPT }\end{array}$ & $\begin{array}{c}13^{\text {th }} \\
\text { OCT }\end{array}$ & $\begin{array}{c}2^{\text {nd }} \\
\text { DEC }\end{array}$ & $\begin{array}{c}13^{\text {th }} \\
\text { JAN }\end{array}$ & $A V R$ \\
\hline 1 & Naucleadiderrichi & 8.40 & 21.02 & 15.66 & 14.25 & 14.83 \\
\hline 2 & Terminaliacatapa & 42.91 & 40.68 & 32.51 & 29.84 & 36.49 \\
\hline 3 & Gmelinaaborea & 4.22 & 3.87 & 3.26 & 3.73 & 3.77 \\
\hline 4 & Khayagrandiofolia & 20.76 & 41.40 & 34.69 & 35.54 & 33.10 \\
\hline 5 & Tectonagrandis & 29.03 & 35.83 & 23.72 & 22.95 & 27.88 \\
\hline 6 & Gambayaalbidum & 19.01 & 32.68 & 19.33 & 17.90 & 22.23 \\
\hline 7 & Cedralaodurata & 16.89 & 18.25 & 13.76 & 13.68 & 15.65 \\
\hline 8 & Triploclantinscheroxylon & 22.63 & 19.47 & 16.12 & 15.80 & 18.51 \\
\hline 9 & Termilinaivorensis & 6.06 & 7.69 & 3.08 & 2.11 & 4.74 \\
\hline 10 & Garcina kola & 12.75 & 3.80 & 2.83 & 1.97 & 5.34 \\
\hline
\end{tabular}

Table 8: Carotenoid $(\mathrm{x}+\mathrm{c})$ values of tree speciesfrom September, 2014 to January 2015 from UnibenForest Reserve.

\begin{tabular}{|c|c|c|c|c|c|c|}
\hline S/N & $\begin{array}{c}\text { CAROTENOID }(x+c) \\
\text { Trees Species }\end{array}$ & $\begin{array}{c}19^{\text {th }} \\
\text { SEPT }\end{array}$ & $\begin{array}{c}20^{\text {th }} \\
\text { OCT }\end{array}$ & $\begin{array}{c}\text { s }^{\text {th }} \\
\text { DEC }\end{array}$ & $\begin{array}{c}20^{\text {th }} \\
\text { JAN }\end{array}$ & AVR \\
\hline 1 & Naucleadiderrichi & 1.07 & 2.56 & 2.09 & 2.04 & 1.94 \\
\hline 2 & Terminaliacatapa & -1.42 & 1.73 & 1.45 & 0.43 & 0.55 \\
\hline 3 & Gmelinaaborea & 5.95 & 2.42 & 1.73 & 2.37 & 3.12 \\
\hline 4 & Khayagrandiofolia & 10.05 & 3.61 & 3.55 & 4.27 & 5.37 \\
\hline 5 & Tectonagrandis & 100.21 & 10.95 & 10.40 & -3.33 & 29.56 \\
\hline 6 & Gambayaalbidum & 76.13 & -2.40 & -2.28 & -1.30 & 17.54 \\
\hline 7 & Cedralaodurata & 6.33 & 2.28 & 1.26 & 1.03 & 2.72 \\
\hline 8 & Triploclantinscheroxylon & 3.90 & 3.15 & 3.11 & 2.20 & 3.09 \\
\hline 9 & Termilinaivorensis & 23.97 & 7.95 & 6.35 & -0.33 & 9.49 \\
\hline 10 & Garcina kola & 14.59 & 2.10 & 1.00 & -0.68 & 4.25 \\
\hline
\end{tabular}

Table 9: Carotenoid $(\mathrm{x}+\mathrm{c})$ values of tree species from September, 2014 to January 2015 from Saponba Forest Reserve.

\begin{tabular}{|c|c|c|c|c|c|c|}
\hline S/N & $\begin{array}{c}\text { CAROTENOID } \\
(x+c) \\
\text { Trees Species }\end{array}$ & $26^{\text {th }}$ SEPT & $27^{\text {th }}$ OCT & $16^{\text {th }}$ DEC & $27^{\text {th }}$ JAN & AVR \\
\hline 1 & Naucleadiderrichi & 9.84 & -1.12 & -0.94 & -0.57 & 1.80 \\
\hline 2 & Terminaliacatapa & 19.64 & 0.38 & 0.52 & 0.39 & 5.23 \\
\hline 3 & Gmelinaaborea & 10.57 & 3.33 & 3.82 & 3.87 & 5.40 \\
\hline 4 & Khayagrandiofolia & 31.12 & 3.59 & 3.73 & 4.11 & 10.63 \\
\hline 5 & Tectonagrandis & 46.97 & 13.84 & 14.28 & 9.97 & 21.26 \\
\hline 6 & Gambayaalbidum & 32.17 & 11.35 & 10.86 & 14.61 & 17.25 \\
\hline 7 & Cedralaodurata & 25.42 & 18.48 & 17.52 & 15.45 & 19.22 \\
\hline 8 & Triploclantinscheroxylon & 1.39 & 1.91 & 1.20 & 0.85 & 1.34 \\
\hline 9 & Termilinaivorensis & -10.56 & 14.69 & 13.99 & 13.05 & 7.79 \\
\hline 10 & Garcina kola & 2.29 & 2.10 & 0.89 & 0.71 & 1.50 \\
\hline
\end{tabular}

\section{Discussion}

Chlorophyll a; In Ogba Forest Reserve, all the trees considered showed a negative response to ambient air pollution except Khayagrandiofolia and Naucleadiderrichi that systematically increased from 20.76 and $8.40 \mathrm{mg} / 100 \mathrm{~g}$ to an average of 33.10 and $14.83 \mathrm{mg} / 100 \mathrm{~g}$ respectively. Garcina Kola has the highest average reduction,, from 12.75 to $5.34 \mathrm{mg} / 100 \mathrm{~g}$. While in UNIBEN forest reserve, apart from species: Naucleadiderrichi and Terminaliacatapa that showed positive variance, the rest of the species showed a drastic content reduction of chlorophyll a, which was

Volume 5 Issue 1, January 2016 


\section{International Journal of Science and Research (IJSR) \\ ISSN (Online): 2319-7064}

Index Copernicus Value (2013): 6.14 | Impact Factor (2014): 5.611

highest in Tectonagrandis: from 100.21 to $29.56 \mathrm{mg} / 100 \mathrm{~g}$ on average. This is over a 3-fold reduction, making it to be a high variation that endangers the plant and biosphere. And in Sakponba forest reserve, only Terminaliaivorensis experienced increase in chlorophyll a, from a negative value $(-10.56)$ to a positive value(average of $7.79 \mathrm{mg} / 100 \mathrm{~g}$ ). The rest tree species show high significant variance, as their chlorophyll a content depleted significantly across the selected period. Suffice to note that Naucleadiderrichi experienced the highest reduction of over 5-fold compared to others.

Chlorophyll b: In Ogba Forest reserve, results showed that six out of ten species: Terminaliacatapa(19.30$30.18 \mathrm{mg} / 100 \mathrm{~g})$, Tectonagrandis $\quad(20.92-84.66 \mathrm{mg} / 100 \mathrm{~g})$, Gambayaalbidum(14.73-21.86mg/100g),

TriplochitonScleroxlon (16.59-20.15mg/100g),

Termilinaivorensis $(20.50-23.68 \mathrm{mg} / 100 \mathrm{~g})$ and Garcina kola (23.93-25.85mg/100g) showed positive variances except Naucleadiderrichi, Gmelinaaborea, Khayagrandiofolia and Cedralaodurata. Suffice to note that Chlorophyll b content depletion in these species was not very significant except Khayagrandiofolia with a 1.4-fold depletion. While in Uniben forest Reseve, variations in chlorophyll b content across the species considered were quite remarkable in terms of reduction in chlorophyll b content, except in Gambayaalbidum species with a positive variance from 19.25 to $20.81 \mathrm{~m} / 100 \mathrm{~g}$. The rest trees experienced a negative variance which was highest in Tectonagrandis from 72.70 to $29.29 \mathrm{mg} / 100 \mathrm{~g}$. Making it to a $2 /$ fold reduction. This is a trend that is similar to Chlorophyll a. In saponba forest reserve, chlorophyll $b$ in all the species considered followed the same trend, as the value decreased across all the species, However Terminaliaivorensis experienced the highest variation of over 2-fold, as chlorophyll b content decreased from 70.33 to $27.83 \mathrm{mg} / 100 \mathrm{~g}$. This is a reduction fold that is similar to that of Uniben Forest reserve. It is however, lowest at Saponba Forest Reserve.

Carotenoid: Ogba forest reserve, an irregular pattern was witnessed in the carotenoid content across the species considered. While Terminaliacatapa, Khayagrandiofolia and Gambayaalbidum, experienced increase in carotenoid contents over the selected or controlled period. The remaining six species experienced decrease in carotenoid content.Although the carotenoid content variations are not very significant across the species $(<2$-fold positive or negativevariance) except Garcina kola with over two fold decrease (negative variance). While UNIBEN forest Reserve, results from the table showed a significant variation in carotenoid content, particularly Tectonagrandisand Gambayaalbidum, from 100.21 to $29.36 \mathrm{mg} / 100 \mathrm{~g}$ and from 76.13 to $17.54 \mathrm{mg} / 100 \mathrm{~g}$ respectively, with the latter having over a 4-fold increase in carotenoid level as against the former (over 3-fold). The rest species experienced reduced carotenoid levels, which were quiet remarkable than those of Ogba forest reserve. And in Saponba forest reserve, variations in carotenoid contents were a drift to those of its counterparts. While Terminaliacatapa has a positive variance in carotenoid content $(-10.56$ to $7.79 \mathrm{mg} / 100 \mathrm{~g})$, the rest species had a negative variance. Suffice to note that from the results,
UNIBEN forest reserve is richer in biomarkers than it counterparts.

The mean values of Chlorophyll-a examined within the three locations are in the sequence

of Khayagrandiolofolia $<$ Triploclantinscheroxylon $<$ Terminal iacatapa $<$ Gambayaalbidium $<$ Garcina

kola $<$ Tectonagrandis $<$ Terminaliaivorensis $<$ Cedralaodurat $a<$ Gmelinaaborea $<$ Naucleadiderrichi.

The mean values of Chlorophyll-b examined within the three locations are in the sequence of

Naucleadiderrichi $<$ Gmelinaaborea $<$ TriploclantinScheroxyl on $<$ Cedralaodurata $<$ Gambayaalbidum $<$ Khayagrandiofolia $<$ Garcina kola $<$ Termilinaivorensis $<$ Tectonagrandis.

The mean values of Carotenoid $(\mathrm{x}+\mathrm{c})$ examined within the three locations are in the sequence of Garcina kola $<$ Gmelinaaborea $<$ Naucleadiderrichi $<$ Termilinaivorens is $<$ Triplochlantinscheroxlon $<$ Cedralaodurata $<$ Terminaliac atapa $<$ Khayagrandiofolia $<$ Gambayaalbidum $<$ Tectonagran dis

\section{Statistical Analysis}

The data collected from the month of September 2014 to January 2015 where subjected to statistical analysis using the SPSS windows 16.0. Below are the results:

Table 10: Results of Anova for Chlorophyll a (Ch-a)

\begin{tabular}{|c|c|c|c|c|c|c|}
\hline \multicolumn{7}{|c|}{ ANOVA } \\
\hline & & $\begin{array}{l}\text { Sum of } \\
\text { Squares }\end{array}$ & Df & $\begin{array}{l}\text { Mean } \\
\text { Square }\end{array}$ & $\mathrm{F}$ & Sig. \\
\hline \multirow{3}{*}{$\begin{array}{c}\text { CHL } \\
\text { A(Ch-a) }\end{array}$} & $\begin{array}{c}\text { Between } \\
\text { Groups }\end{array}$ & 294.101 & 2 & 147.05 & 1.152 & 0.331 \\
\hline & $\begin{array}{l}\text { Within } \\
\text { Groups }\end{array}$ & 3447.008 & 27 & 127.667 & & \\
\hline & Total & 3741.109 & 29 & & & \\
\hline
\end{tabular}

Table 11: Results of Anova for Chlorophyllb(Ch-b)

\begin{tabular}{|c|c|c|c|c|c|c|}
\hline \multicolumn{7}{|c|}{ ANOVA } \\
\hline & & $\begin{array}{l}\text { Sum of } \\
\text { Squares }\end{array}$ & $\mathrm{df}$ & $\begin{array}{l}\text { Mean } \\
\text { Square }\end{array}$ & $\mathrm{F}$ & Sig. \\
\hline \multirow{3}{*}{$\begin{array}{l}\text { CHLB } \\
\text { (Ch-b) }\end{array}$} & $\begin{array}{c}\text { Between } \\
\text { Groups }\end{array}$ & 535.07 & 2 & 267.535 & 1.526 & 0.236 \\
\hline & $\begin{array}{l}\text { Within } \\
\text { Groups }\end{array}$ & 4734.662 & 27 & 175.358 & & \\
\hline & Total & 5269.732 & 29 & & & \\
\hline
\end{tabular}

Table 12: Results for Anova for Carotenoid $(x+c)$

\begin{tabular}{|c|c|c|c|c|c|c|}
\hline \multicolumn{7}{|c|}{ ANOVA } \\
\hline \multirow{2}{*}{$\begin{array}{c}\text { Sum of } \\
\text { Squares }\end{array}$} & Df & $\begin{array}{c}\text { Mean } \\
\text { Square }\end{array}$ & F & Sig. \\
\hline $\begin{array}{c}\text { C } \\
(\mathrm{x}+\mathrm{c})\end{array}$ & Between Groups & 649.971 & 2 & 324.985 & 3.501 & 0.044 \\
\cline { 2 - 8 } & Within Groups & 2506.366 & 27 & 92.828 & & \\
\cline { 2 - 8 } & Total & 3156.337 & 29 & & & \\
\hline
\end{tabular}

Tables 10.11.12 show that there was no significant difference between the three locations among the 10 tree species during the study period using one-way ANOVA. But the values of chlorophyll and carotenoids reduced from raining season to dry season. 
International Journal of Science and Research (IJSR)

ISSN (Online): 2319-7064

Index Copernicus Value (2013): 6.14 | Impact Factor (2014): 5.611

\section{Trend Diagrams}

The trend diagrams are used to the show the trend of values of Chlorophyll a, Chlorophyll b and Carotenoid content within the four months study period.

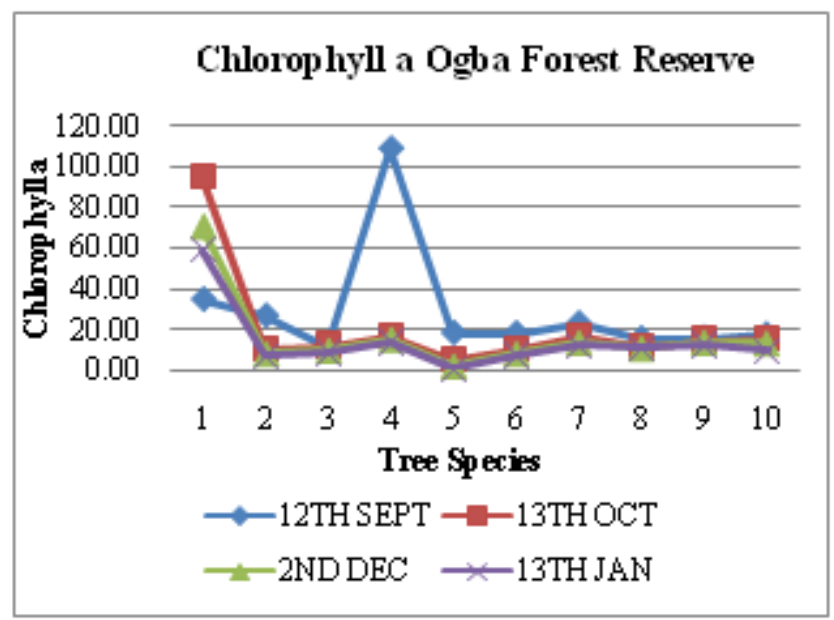

Figure 2: Ch-a Saponba Forest Reserve

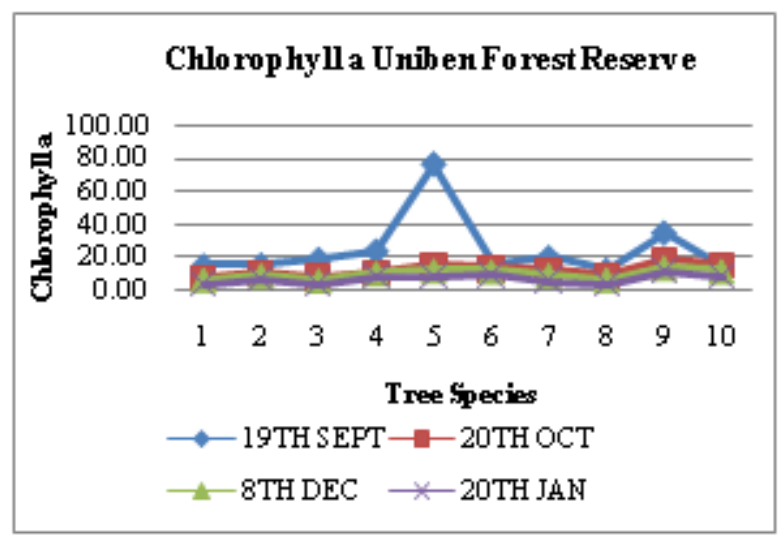

Figure 3: Ch-a UNIBEN Forest Reserve

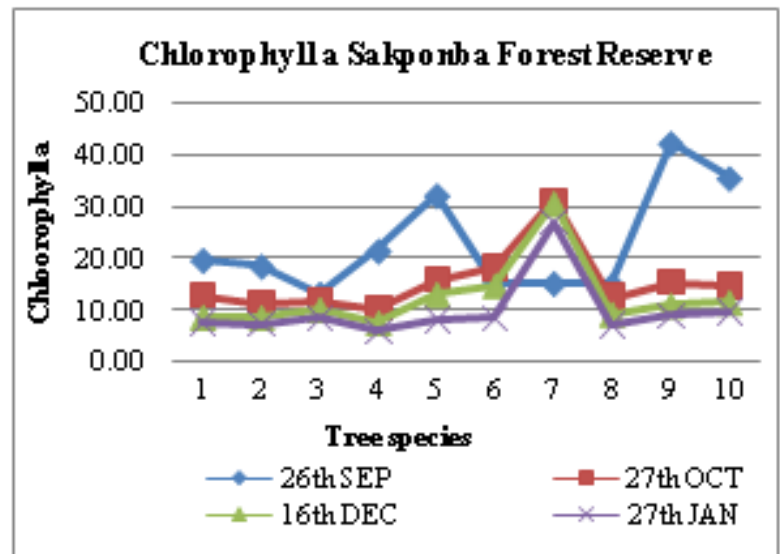

Figure 4: Ch-a Sakponba Forest Reserve

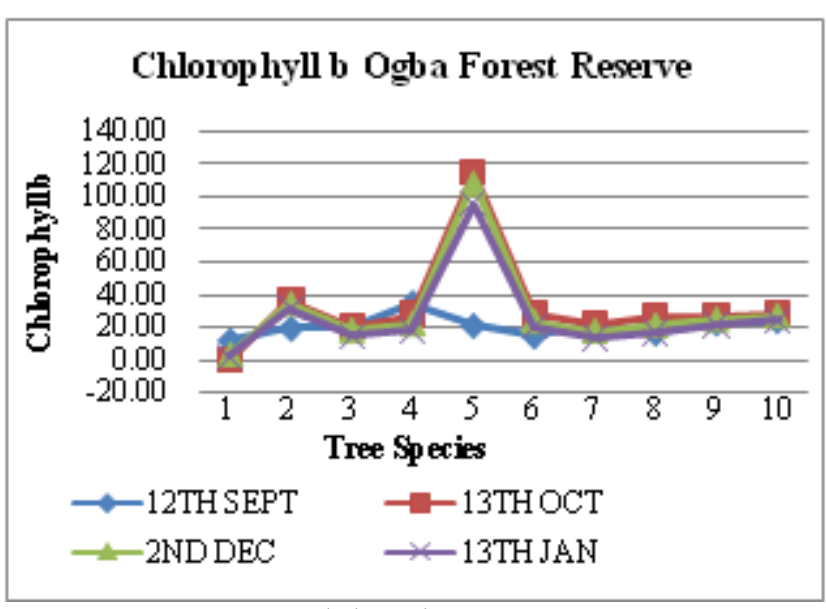

Figure 5: Ch-b Ogba Forest Reserve

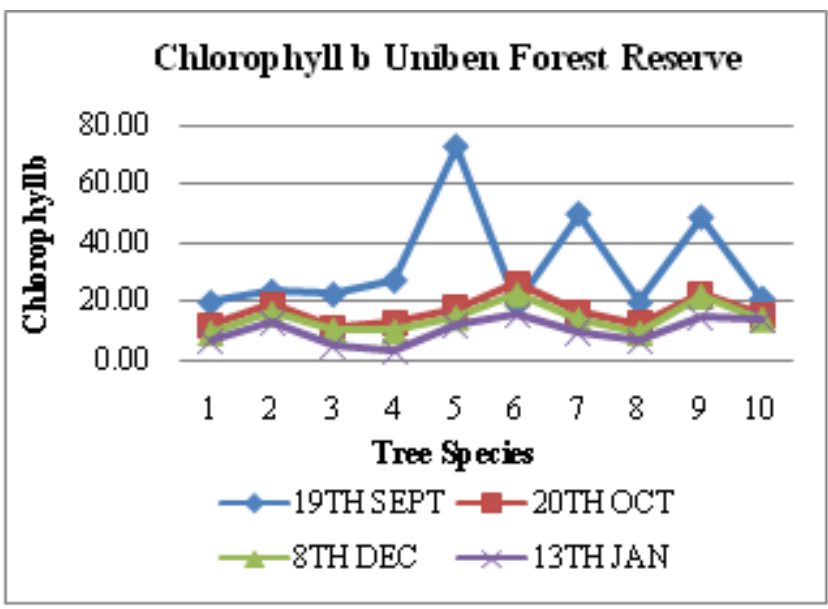

Figure 6: Ch-b UNIBEN forest Reserve

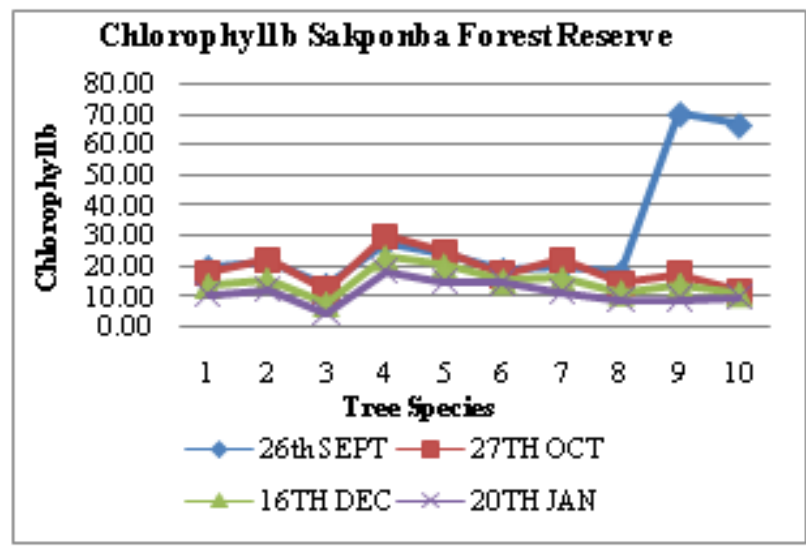

Figure 7: Ch-b forSaponba Forest Reserve

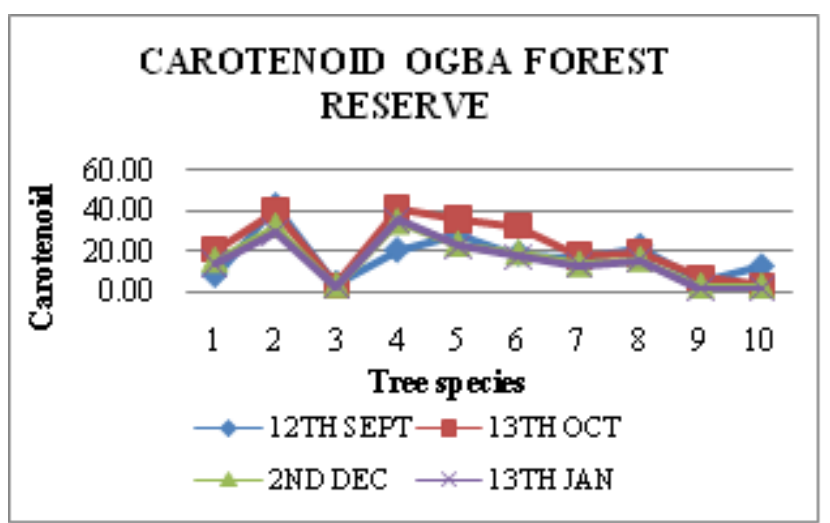

Figure 8: Carotenoid $(\mathrm{x}+\mathrm{c})$ forOgba Forest Reserve

Volume 5 Issue 1, January 2016 


\section{International Journal of Science and Research (IJSR) \\ ISSN (Online): 2319-7064}

Index Copernicus Value (2013): 6.14 | Impact Factor (2014): 5.611

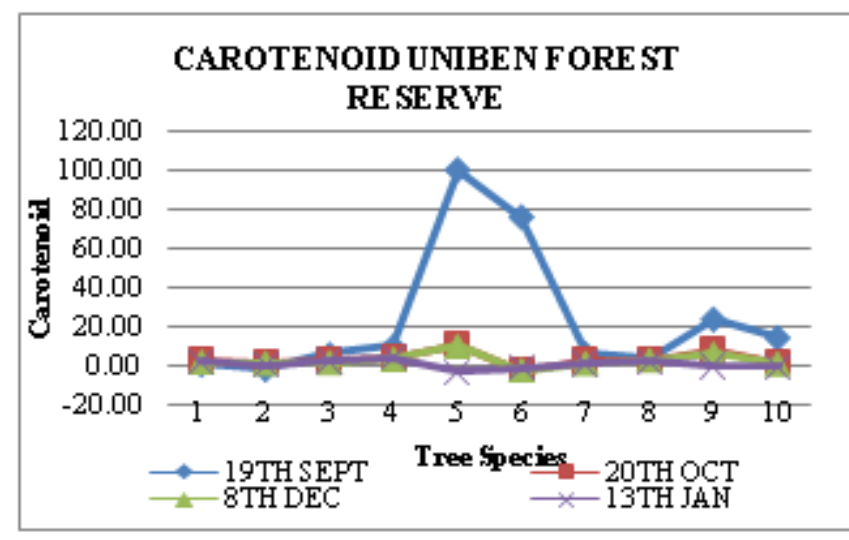

Figure 9: Carotenoid $(\mathrm{x}+\mathrm{c})$ for UNIBEN forest Reserve

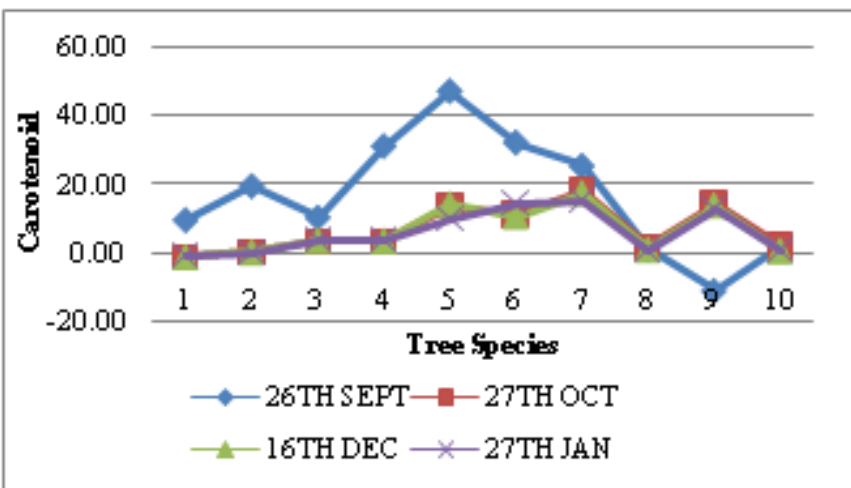

Figure 10: Carotenoid $(\mathrm{x}+\mathrm{c})$ for Saponba Forest Reserve

\section{Conclusion}

Evaluating the levels of chlorophyll-a, Chlorophyll-b and Carotenoid in 3 artificial forests in Edo state(Ogba, UNIBEN and Saponba Forest Reserves), Nigeria from selected months in the raining season till the dry season in order to access their responses to ambient air pollutants, notable variations occurred as Terminaliacatapa had the highest value of chlorophyll a content with a value of $36.49 \mathrm{mg} / 100 \mathrm{~g}$ in Ogba Forest Reserve while the lowest value was also in Terminaliacatapa with a value of $0.55 \mathrm{mg} / 100 \mathrm{~g}$ in uniben forest reserve. Chlorophyll b content was highest in Tectonagrandis in the Ogba Forest Reserve of a value of $84.66 \mathrm{mg} / 100 \mathrm{~g}$ and lowest in a value of $4.15 \mathrm{mg} / 100 \mathrm{~g}$ in Naucleadiderrichi also in the Ogba Forest Reserve. Carotenoid $(\mathrm{x}+\mathrm{c})$ content was highest in Terminaliacatapa with a value of $36.49 \mathrm{mg} / 100 \mathrm{~g}$ and lowest in UNIBEN Forest reserve with a value of $0.55 \mathrm{mg} / 100 \mathrm{~g}$.

\section{References}

[1] Agbaire, P.O and Esiefarienrhe, E. (2009): Air pollution tolerance indices (APTI) of some plants around Otorogun Gas Plant In Delta State, Nigeria. Journal of Applied science environmental management. 13:11-14

[2] Bauernfeind, J.C (1981): Carotenoid as colorants and vitamin A precursors. Academic Press, New York.

[3] Ceulemans, R. \&Saugier, B. (1993): Photosynthesis. In: Ragha-Vendra, A.S (Ed). Physiology of Trees. New York: John Wiley \& son: p.21-50

[4] Demming-Adams, B., Adams III, W.W (1992): Role of xanthophlls cycle carotenoids in the protection of photosynthesis. Trend in plant science, Vol. 1:21-26
[5] Evans J and Turnbull, J.W (2004): Plantation Forestry in the Tropics. $3^{\text {rd }}$ Edition, Oxford University Press,London

[6] FRIN (1977): www.frin.gov.ng

[7] Gamon, J.A and Surfus, J.S (1999): Assessing leaf pigment content and activity with a reflectometer. New Phytologist 143, 105-117.

[8] Iyawe J.G. (1989): The ecology of small mammals in Ogba Forest Reserve, Nigeria. Journal of Tropical Ecology 5(1):5164

[9] James A.O. and Akaranta O(2001):Inhibition of Zinc in Hydrochlororic acid solution by Red Onion Skin Acetoneextract, Res. J. Chem. Sci., 1(1), 31-37

[10]Lichtenthaler, H. K. 1987. Chlorophylls and carotenoids: pigments of photosynthetic biomembranes. Methods Enzymol, 148: 350-382.

[11] Lichtenthaler, H. K. \&Buscher, C. 2001. Chlorophylls and carotenoids: measurement and characterization by UV-VIS spectroscopy. Curr.Protoc.Food Anal. Chem., F: 1-8.

[12] Lisiewska, Z., S"upski, J., Korus, A., 2001. Influence of cultivationperiod, cultivar and usable part on content of chlorophylls andvolatile oils in dill (Anethumgraveolens L.). Electronic Journal of Polish Agricultural Universites.Food Science and Technology http://www.ejpau.media.pl/series/volume4/issue2/food/art18.html.

[13] Nichiporovich, A.A. (1974): Chlorophyll and Photosynthetic productivity in plants. Shlyk, A.A., Ed., Minsk: Nauka I Tekhnika, p 49-62

[14] Porra, R. J. (1991): Recent advances and re-assessments inchlorophyll extraction and assay procedures for terrestrial, aquatic, and marine organisms, including recalcitrant algae.In: Scheer H (ed) Chlorophylls, 31-57 (1991)

[15] Richardson, A.D., Duigan, S.P and Berlyn, G.P (2002): An evaluation of noninvasive methods of estimate foliar chlorophyll content. J.Arid Environ., 75:185-194

[16] Ruiz-Jaen Maria C. and Mitchel Aide (2005): Restoration Ecology. 13(3):569-577

[17] Siefermann-Harms, D. (1987): The light harvesting and protective function of carotenoids in photosynthetic membranes. PhysiologiaPlantarum, 69:561-568

[18] Tripathi A. K. and Gautam M., Biochemical parameters of plants as indicators of air Pollution, J. Environ. Biol., 28, 127132 (2007)

[19] University of Benin Master plan (1993): university press pp79:21

[20] UsuanleleI.N(1999): The effect of forest management on soils physical and chemical properties and floristic composition(A case study of university of Benin Forest Plantation and Adjacent Natural Forest) undergraduate Dissertation, Dept of Forestry and Wildlife, University of Benin in, Benin-city, Edo State, Nigeria. Pp63

[21] Vicas .S,Rugina, D and C. Sosaciu (2008): Antioxidant activities of Viscum album leaves from various host trees. Bulletin UASVM, Agriculture Cluj-Napoca 65:327-332

[22] Wellburn A. R (1994): The spectral determination of chlorophylls a and $\mathrm{b}$, as well as total carotenoids, using various solvents with spectrophotometers of different resolution, J. Plant Physiol., 144, 307-313

[23] Young, A. and Britton, G (1993): Carotenoid in Photosynthesis, $1^{\mathrm{ST}}$ Edition. Chapman and Hall, London. Pp 498

[24] Zarco-Tejada, P.J, Miller,JR., Mohammed, G.H,. Noland, TL and Sampson, P.H (2002): Vegetation stress detection through chlorophyll $\mathrm{a}+\mathrm{b}$ estimation and fluorescence effects on hyperspectral imagery. Journal of Environmental Quality. 31:1433-1441 